\title{
Acidente vascular encefálico isquêmico devido à síndrome do anticorpo antifosfolípide em paciente lúpica - um relato de caso
}

\author{
Ischemic stroke due to antiphospholipid syndrome in a lupus patient - a case report \\ Thiago Azevedo de Lucena Costa ${ }^{1}$ (D), Leonardo Fernandes e Santana ${ }^{1}$ (D), Mateus de Sousa Rodrigues ${ }^{1}$ (D), \\ Caroline Diniz Bezerra2 (D), Orlando Vieira Gomes ${ }^{3}$ (D), Rita Marina Soares de Castro Duarte ${ }^{4}$ (D)
}

\begin{abstract}
RESUMO
Lúpus eritematoso sistêmico é uma doença de origem autoimune que apresenta um amplo espectro de manifestações clínicas causadas pelo estado inflamatório crônico. A associação com a síndrome do anticorpo antifosfolípide ocorre em aproximadamente $36 \%$ dos pacientes lúpicos. Nesses pacientes, a doença de base e o uso crônico de corticosteroides contribuem para aterosclerose acelerada, uma complicação que agrega risco cardiovascular. Relatamos um caso de acidente vascular encefálico em paciente de 25 anos portadora de lúpus eritematoso sistêmico e síndrome do anticorpo antifosfolípide.
\end{abstract}

Palavras-chave: Acidente Vascular Cerebral; Lúpus Eritematoso Sistêmico; Síndrome Antifosfolipídica.

\begin{abstract}
Systemic lupus erythematosus is an autoimmune disease that presents a wide spectrum of clinical manifestations caused by the chronic inflammatory state. Association with antiphospholipid antibody syndrome occurs in approximately $36 \%$ of lupus patients. In these patients, the underlying disease and the chronic use of corticosteroids contribute to accelerated atherosclerosis, a complication that adds increased cardiovascular risk. We report a case of stroke in a 25-year-old patient with systemic lupus erythematosus and antiphospholipid antibody syndrome.
\end{abstract}

Keywords: Stroke; Systemic Lupus Erythematosus; Antiphospholipid Syndrome.

1. Discente de Medicina na Universidade Federal do Vale do São Francisco (UNIVASF), Petrolina (PE), Brasil.

2. Médica Residente de Clínica Médica do Hospital Universitário da UNIVASF, Petrolina (PE), Brasil.

3. Médico Nefrologista. Preceptor da Residência Médica de Clínica Médica do Hospital Universitário da UNIVASF. Professor MSC efetivo da Disciplina de Medicina de Urgência da UNIVASF, Petrolina (PE), Brasil.

4. Médica Reumatologista. Preceptora da Residência Médica de Clínica Médica do Hospital Universitário da UNIVASF. Professora efetiva da Disciplina de Reumatologia da UNIVASF, Petrolina (PE), Brasil

$\triangle$ Thiago Azevedo de Lucena Costa. Av. José de Sá Maniçoba. CEP: 56304-917. Petrolina (PE), Brasil. thiagoalcosta123@gmail.com | Recebido em: 08/11/2019 | Aprovado em: 17/07/2020 


\section{INTRODUÇÃO}

O acidente vascular encefálico (AVE) está entre as maiores causas de morbimortalidade no mundo todo ${ }^{1}$. Seu impacto negativo vai além do indivíduo afetado, contribuindo com uma parcela significativa do orçamento voltado para a saúde no Estado. Menos de $10 \%$ dos casos acontecem em indivíduos abaixo dos 45 anos de idade 2 . Nesse grupo populacional, trombofilias adquiridas e doenças autoimunes costumam ser os principais fatores precipitantes, sendo o Lúpus Eritematoso Sistêmico (LES) uma causa importante, por se tratar de uma doença inflamatória sistêmica crônica². Merece destaque a Síndrome do Anticorpo Antifosfolipídio (SAAF), responsável por mais de $20 \%$ dos AVEs em indivíduos com menos de 45 anos de idade ${ }^{3-7}$.

\section{RELATO}

Paciente do sexo feminino, 25 anos, trazida ao serviço de emergência após surgimento de cefaleia intensa, vômitos, perda de força em dimídio direito e disartria há 72 horas. 0 exame físico admissional evidenciou déficit motor à direita, disartria, artrite em pequenas articulações (metacarpofalangeanas e interfalangeanas proximais bilateralmente) e púrpuras em membros inferiores, sem outras lesões de pele evidentes ou relato de fotofobia. Apresentou passado de epilepsia e de hipertensão arterial sistêmica. Negou história médica pregressa semelhante, outras comorbidades ou uso de drogas lícitas e ilícitas. O antecedente obstétrico é marcado por três abortos prévios, um na $3^{a}$ e dois na $8^{a}$ semana de gestação. A tomografia computadorizada (TC) de crânio (Figura 1) evidenciou área de hipodensidade frontoparietal à esquerda aguda/subaguda, sem sinais de sangramento ou desvio da linha média. Os demais exames admissionais estão descritos na Tabela 1.

Após avaliação inicial, com exclusão de etiologias virais envolvidas no diagnóstico diferencial, foi estabelecido o diagnóstico de LES, com o auxílio dos critérios classificatórios de 2012, publicados pela Systemic Lupus International Collaborating Clinics (SLICC) ${ }^{8}$, dos quais a paciente apresentava: (1) Fator Antinuclear (FAN), (2) sinovite de duas ou mais articulações, (3) proteinúria/24h $>500 \mathrm{mg}$,
(4) anti-DNAds, (5) complemento consumido e (6) perfil antifosfolipídico triplamente positivo, (7) trombocitopenia. A história de abortos de repetição, trombose arterial e a presença de perfil antifosfolípide triplamente positivo (anticoagulante lúpico, anticorpo anticardiolipina IgM e anticorpo anti-beta2 glicoproteína 1 IgG reagentes) sugeriu a presença de SAAF. Esta entidade foi confirmada após novos registros de anticorpos antifosfolipídeos positivos em 12 semanas.

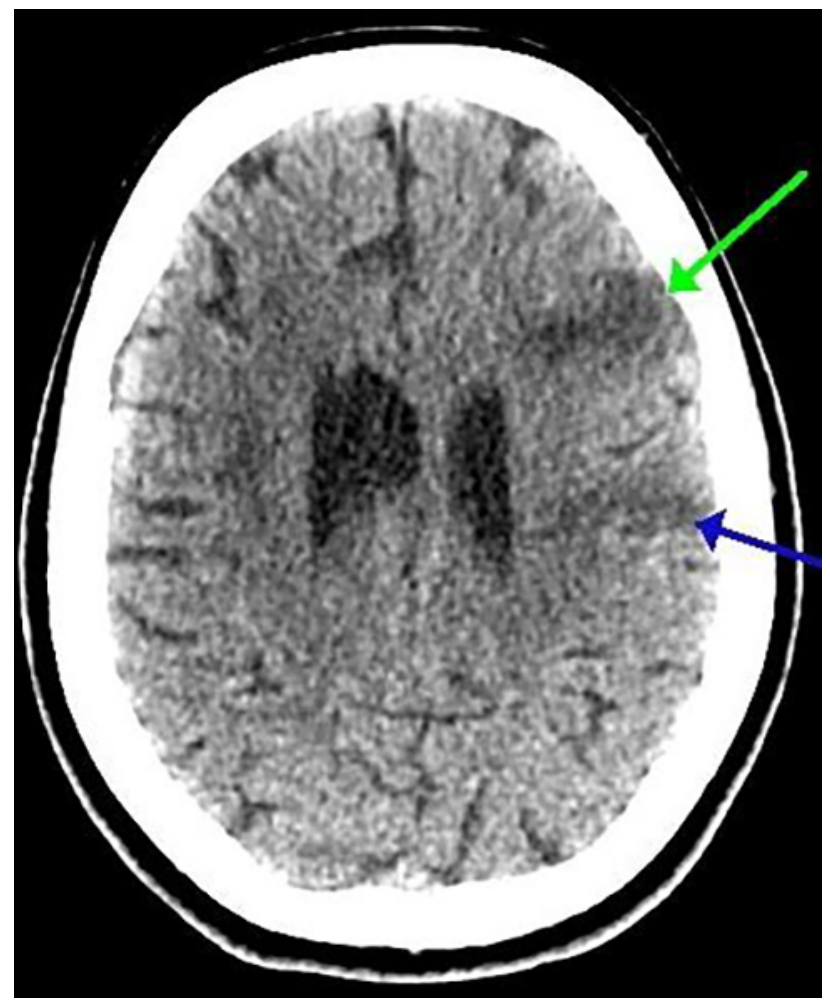

Figura 1: Tomografia computadorizada de crânio. As setas evidenciam área de hipodensidade frontoparietal esquerda alteração sugestiva de AVE isquêmico.

O evento cerebrovascular foi conduzido com anticoagulação e antiagregação plaquetária. Devido à alta atividade do LES, quantificada pelo Systemic Lupus Erythematosus Disease Activity Index 2000 (SLEDAI-2K), escore utilizado para avaliar a atividade de doença no LES, no qual a paciente apresentou um escore de 25 , foi indicada a pulsoterapia com metilprednisolona por via intravenosa $1 \mathrm{~g} / \mathrm{dia}$, durante três dias consecutivos. A paciente evoluiu com melhora clínica, em condições de alta hospitalar e de acompanhamento ambulatorial. 
Tabela 1

Exames laboratoriais admissionais

\begin{tabular}{|c|c|c|c|}
\hline Exame & Valor & Exame & Valor \\
\hline Hemoglobina & $\begin{array}{c}10,5 \mathrm{~g} / \mathrm{dL} \\
\text { [valor de referência: } 12,0-15,0 \mathrm{~g} / \mathrm{dL} \text { ] }\end{array}$ & Anti-SM & Não reagente \\
\hline Hematócrito & $\begin{array}{c}30,8 \% \\
\text { [valor de referência: } 36-46 \%]\end{array}$ & Sumário de Urina & $\begin{array}{c}\text { Ausência de cilindros celulares e de } \\
\text { hematúria }\end{array}$ \\
\hline $\begin{array}{l}\text { Leucócitos } \\
\text { Linfócitos }\end{array}$ & $\begin{array}{c}5310 / \mathrm{mm}^{3} \\
\text { [valor de referência: } 4000-10000 / \mathrm{mm}^{3} \text { ] } \\
1221 / \mathrm{mm}^{3} \\
\text { [valor de referência: } 1000-3000 / \mathrm{mm}^{3} \text { ] }\end{array}$ & $\begin{array}{l}\text { Teste de Coombs } \\
\text { direto }\end{array}$ & Não reagente \\
\hline Plaquetas & $\begin{array}{c}54000 / \mathrm{mm}^{3} \\
\text { [valor de referência: } \\
\left.150.000-450.000 / \mathrm{mm}^{3}\right]\end{array}$ & P-ANCA & Não reagente \\
\hline Ureia & $\begin{array}{c}74 \mathrm{mg} / \mathrm{dL} \\
\text { [ valor de referência: } 16-40 \mathrm{mg} / \mathrm{dL} \text { ] }\end{array}$ & C-ANCA & Não reagente \\
\hline Creatinina & $\begin{array}{c}0,9 \mathrm{mg} / \mathrm{dL} \\
\text { [valor de referência: } 0,5-1,1 \mathrm{mg} / \mathrm{dL} \text { ] }\end{array}$ & $\begin{array}{l}\text { Relação Proteína/ } \\
\text { Creatinina urinária }\end{array}$ & $\begin{array}{c}2,3 \\
\text { [valor de referência: }<0,2]\end{array}$ \\
\hline $\begin{array}{l}\text { FAN } \\
\text { (Fator antinuclear) }\end{array}$ & $\begin{array}{l}\text { Reagente - padrão nuclear homogêneo } \\
(1: 640)\end{array}$ & Proteinúria de $24 \mathrm{~h}$ & $\begin{array}{c}893 \mathrm{mg} / 24 \mathrm{~h} \\
\text { [valor de referência: }<150 \mathrm{mg} / 24 \mathrm{~h}]\end{array}$ \\
\hline Anti-DNAds & $\begin{array}{l}\text { Reagente } \\
(1: 160)\end{array}$ & $\mathrm{C} 3$ & $\begin{array}{c}2,2 \mathrm{mg} / \mathrm{dL} \\
\text { [valor de referência: } 90-180 \mathrm{mg} / \mathrm{dL} \text { ] }\end{array}$ \\
\hline $\begin{array}{l}\text { Anticoagulante } \\
\text { lúpico }\end{array}$ & Reagente & $\mathrm{C} 4$ & $\begin{array}{c}71,1 \mathrm{mg} / \mathrm{dL} \\
\text { [valor de referência: } 16-38 \mathrm{mg} / \mathrm{dL}]\end{array}$ \\
\hline Anticardiolipina IgM & $\begin{array}{c}\text { Reagente }(81,3) \\
{[\text { valor de referência: < 10] }}\end{array}$ & PCR & $\begin{array}{c}65 \mathrm{mg} / \mathrm{L} \\
\text { [ valor de referência: }<3,0 \mathrm{mg} / \mathrm{L}]\end{array}$ \\
\hline $\begin{array}{l}\text { Anti-B2GP1 IgG } \\
\text { (anti-beta-2 } \\
\text { glicoproteína 1) }\end{array}$ & $\begin{array}{l}\text { Reagente }(64) \\
\text { [valor de referência: < 5] }\end{array}$ & VHS & $\frac{145 \mathrm{~mm}}{\text { [valor de referência }<20 \mathrm{~mm} / \mathrm{h} \text { ] }}$ \\
\hline Anti-SSA/Anti-SSB & Não reagentes & $\begin{array}{l}\text { Sorologias para } \\
\text { citomegalovírus, } \\
\text { sífilis, Epstein- } \\
\text { Barr, hepatite C, } \\
\text { hepatite B e HIV }\end{array}$ & Não Reagentes \\
\hline
\end{tabular}

\section{DISCUSSÃO}

O diagnóstico sindrômico de déficit neurológico focal em pacientes jovens, especialmente aqueles com menos de 45 a 50 anos de idade, requer um raciocínio clínico etiológico diferente daquele já consolidado para pacientes com idade mais avançada. A abordagem inicial do AVE isquêmico nesse grupo de pacientes deve envolver a busca ativa por condições externas, drogas ou doenças de base que possam aumentar o risco tromboembólico e cardiovascular ${ }^{2,9}$.

A elucidação do diagnóstico diferencial deve ter início na avaliação dos medicamentos e drogas lícitas/ilícitas utilizadas pelo paciente. Drogas ilícitas (Ex. cocaína, crack) estão entre os grandes responsáveis pelos eventos cerebrovasculares nesses pacientes. Anticoncepcionais orais combinados devem ser lembrados, principalmente naquelas pacientes tabagistas e/ou portadoras de enxaqueca, por apresentarem alto risco trombogênico na vigência concomitante dessas condições ${ }^{9}$.

Após a avaliação inicial dos fatores externos associados ao evento isquêmico cerebrovascular, devem ser lembradas as comorbidades, hereditárias e adquiridas, que também elevam tal risco. No Brasil, a anemia falciforme representa uma importante causa de AVE em pacientes jovens. Trata-se de uma hemoglobinopatia hereditária que confere um aumento da viscosidade sanguínea, consumo de óxido nítrico, lesão endotelial e inflamação crônica, cuja fisiopatologia leva a complicações isquêmicas multiorgânicas, entre as quais se encontra a isquemia cerebral. Desordens menos comuns, como trombofilias hereditárias, devem ser pesquisadas após exclusão de etiologias mais prevalentes ${ }^{9}$.

Inúmeras doenças infecciosas podem cursar com déficit neurológicos focais e devem ser 
prontamente excluídas, entre as quais se destacam as meningoencefalites, a sífilis, a doença associada ao HIV, endocardite infecciosa, neurotoxoplasmose e a neurocisticercose. A abordagem diagnóstica dessas doenças depende da análise sorológica, de culturas, de exames de imagem (por exemplo, tomografia computadorizada, ecocardiograma) e do estudo do líquor ${ }^{9-11}$.

Estigmas de doenças sistêmicas adquiridas, principalmente de natureza autoimune, devem ser avaliados pelo exame físico inicial e complementados pelos exames laboratoriais. A presença de artropatias, miopatias, rash cutâneo, púrpuras, fotossensibilidade, disfunção de glândulas endócrinas e exócrinas múltiplas, insuficiência renal, presença de pressões arteriais diferentes entre os membros, são alguns dos estigmas que devem ser buscados na avaliação inicial desses pacientes, a fim de proporcionar uma investigação mais direcionada da etiologia específica. Entre as principais desordens autoimunes, encontram-se as colagenoses (LES, esclerose sistêmica), vasculites (arterite de Takayasu, vasculite do sistema nervoso central), microangiopatias trombóticas (SAAF, púrpura trombocitopênica trombótica), entre outras $3,5,12$.

O presente relato de caso envolve a presença de duas comorbidades que conferem elevado risco de eventos trombóticos cerebrovasculares. O LES é uma doença autoimune que envolve a produção de autoanticorpos com formação de imunocomplexos e potencial acometimento de qualquer órgão. Por se tratar de uma doença heterogênea, seus critérios classificatórios foram adaptados com o tempo, de modo paralelo aos novos estudos acerca de sua base fisiopatológica. Os critérios classificatórios da SLICC foram elaborados e validados com a proposta de agregar sensibilidade aos primeiros critérios, propostos em 1982, de modo a facilitar o diagnóstico precoce do LES $^{8}$. Dentre esses critérios, merece destaque o perfil antifosfolípide, uma vez que a SAAF apresenta, em cerca de $36 \%$ dos pacientes, associação com o LES, o que motiva sua investigação, principalmente na presença de história pregressa de abortos de repetição ${ }^{12}$. Nesses casos, há grande elevação do risco trombogênico. Os domínios clínicos, renal e musculoesquelético, também foram contemplados. O acometimento renal, importante fator determinante de morbimortalidade, é observado em até $50 \%$ dos pacientes com LES.
Comumente está associado ao anti-DNAds. No caso relatado, a paciente apresentava uma proteinúria acima de $500 \mathrm{mg}$ em 24 horas, achado presente em virtualmente $100 \%$ dos pacientes com nefrite lúpica ${ }^{13}$.

A associação de LES com o aumento do risco de eventos cerebrovasculares já está bem estabelecida, sendo estes eventos responsáveis pelo óbito de 20 a $30 \%$ desses indivíduos. Vários mecanismos estão envolvidos na gênese do AVE nesses casos, dos quais merecem destaque: aterosclerose acelerada e precoce, infiltração perivascular de células inflamatórias e trombose, principalmente quando há associação com a SAAF, devido ao constante estado de hipercoagulabilidade ${ }^{14}$.

\section{CONCLUSÃO}

A SAAF é a principal trombofilia adquirida e deve ser lembrada em pacientes jovens que sofrem tromboses e/ou perdas gestacionais recorrentes. 0 AVE é a complicação arterial mais comum e grave da SAAF $^{5}$. Este caso serve de alerta para o reconhecimento precoce de doenças autoimunes como fatores precipitantes de eventos cerebrovasculares. Se não diagnosticadas e tratadas corretamente, encerram alto risco de recorrência, promovendo altos índices de morbimortalidade ${ }^{15}$.

\section{REFERÊNCIAS}

1. Johnson C, Nguyen M, Roth G, Nichols E, Alam T, Abate $D$, et al. Global, regional, and national burden of stroke, 1990-2016: a systematic analysis for the Global Burden of Disease Study 2016. The Lancet Neurology. 2019;18(5):439-458.

2. Yamamoto, FI. Ischemic stroke in young adults: an overview of etiological aspects. Arquivos de neuro-psiquiatria. 2012:70(6):462-466.

3. Hughes GR. V. Migraine, memory loss, and "multiple sclerosis". Neurological features of the antiphospholipid (Hughes') syndrome. Post grad Med J. 2003. Feb, 79 (928): 81-3

4. Carvalho MAP. Bertolo MB, Lanna CCD. Reumatologia Diagnóstico e Tratamento. Ed Guanabara Koogan, 2014. ISBN 6561141662.

5. Ricarte IF, Dutra LA. Manifestações neurológicas da síndrome do anticorpo antifosfolipídio. Ver Paul Reumatol. 2018 abr-jun; 17(2):12-7. 
6. Smajlović D. Strokes in young adults: epidemiology and prevention. Vasc Health Risk Manag. 2015;11:157-164.

7. Janssen AW, de Leeuw FE, Janssen MC. Risk factors for ischemic stroke and transient ischemic attack in patients under age 50. J Thromb Thrombolysis. 2011;31(1):8591. doi:10.1007/s11239-010-0491-3

8. Petri M, Orbai AM, Alarcon GS, Gordon C, Merrill JT, Fortin PR, et al. Derivation and validation of the Systemic Lupus International Collaborating Clinics classification criteria for systemic lupus erythematosus. Arthritis Rheum 2012. 64: 2677.

9. Ferro JM, Massaro AR, Mas JL. Aetiological diagnosis of ischaemic stroke in young adults. Lancet Neurol. 2010;9(11):1085-1096.

10. Al-Saeed WM, Oleiwi Al-Kuraishi AH, Dahash SL, Al-Gareeb AI, Alkuraishy HM. Neurocysticercosis: A new concept and insight into basic and future pharmacotherapy. J Pak Med Assoc. 2019;69(Suppl 3)(8):S113-S118.
11. Vidal JE. HIV-Related Cerebral Toxoplasmosis Revisited: Current Concepts and Controversies of an Old Disease. J Int Assoc Provid AIDS Care. 2019;18:2325958219867315.

12. Amorim L, Maia F, Rodrigues C. Stroke in systemic lupus erythematosus and antiphospholipid syndrome: risk factors, clinical manifestations, neuroimaging, and treatment. Lupus. 2017:26(5):529-536.

13. Almaani S, Meara A, Rovin BH. Update on lupus nephritis. Clinical Journal of the American Society of Nephrology. 2017:12(5):825-835.

14. Cavallaro $M$, Barbaro $U$, Caragliano A, Longo $M$, Cicero G, Granata F, et al. Stroke and Systemic Lupus Erithematosus: a review. EMJ Rheumatology. 2018:5(1):100-107.

15. Sanna G, D'Cruz D, Cuadrado MJ. Cerebral manifestations in the antiphospholipid (Hughes') syndrome. Rheum Dis Cli North Am. 2006; 32(3): 465-90. 